\title{
Aptamer based surface plasmon resonance sensor for aflatoxin B1
}

\author{
Linlin Sun ${ }^{1,2} \cdot$ Liqing $\mathrm{Wu}^{3} \cdot$ Qiang Zhao ${ }^{1,2}$
}

Received: 27 March 2017 / Accepted: 11 April 2017/Published online: 19 April 2017

(C) Springer-Verlag Wien 2017

\begin{abstract}
The authors describe a surface plasmon resonance (SPR) based aptasensor for the carcinogenic mycotoxin aflatoxin B1 (AFB1) in a direct assay format. The aptamer is immobilized on the surface of a commercial sensor chip, and the SPR signal increases on binding of AFB1. The sensor chip can be fully regenerated by passing a flow of buffer over it upon which bound AFB1 dissociates from the aptamer. The biosensor works in the $0.4 \mathrm{nM}$ to $200 \mathrm{nM}$ AFB1 concentration range and has a $0.4 \mathrm{nM}$ detection limit. It allows AFB1 to be determined in complex samples such as diluted red wine and beer. The assay is sensitive, and the chip is easily regenerated and stable. The method therefore overcomes certain limitations of antibody-based SPR assays and of competitive SPR assays for AFB1.
\end{abstract}

Keywords Mycotoxin · SPR · Direct format assay · Affinity binding $\cdot$ Antibody $\cdot$ Chip $\cdot$ Regeneration $\cdot$ Immobilization · Dissociation $\cdot$ Stability

Electronic supplementary material The online version of this article (doi:10.1007/s00604-017-2265-5) contains supplementary material, which is available to authorized users.

Qiang Zhao

qiangzhao@rcees.ac.cn

1 State Key Laboratory of Environmental Chemistry and Ecotoxicology, Research Center for Eco-Environmental Sciences, Chinese Academy of Sciences, Beijing 100085, China

2 University of Chinese Academy of Sciences, Beijing 100049, China

3 National Institute of Metrology, Beijing 100029, China

\section{Introduction}

Aflatoxin B1 (AFB1) shows toxicity and carcinogenic effects on human and animals $[1,2]$. Detection of AFB1 is important in food safety, quality control, environmental analysis, and toxin sensing. Currently, the existing methods for AFB1 detection are mainly based on high performance liquid chromatography (HPLC) [3] and immunoassay [4-7]. HPLC coupled with fluorescence detection or mass spectrometry has strength in high accuracy and good repeatability, but it usually needs time-consuming and laborious process. Antibodies based immunosensors are suitable for rapid detection on site. However, the antibodies also meet some limitations in preparation, stability, and cost.

Compared to antibodies, nucleic acid ligand aptamers have unique features, such as ease of chemical synthesis and modification with functional groups, and good stability [8,9]. Aptamers have been used in biosensors and versatile assays for a variety of targets [8-11]. The aptamer for AFB1 has been selected and provides new choices for detection of AFB1 using aptamer as affinity ligand [12]. Several aptamer based sensors and assays for AFB1 have been reported [13-18]. Lu et al. [13] demonstrated an aptamer-based fluorescence detection method for sensitive AFB1 monitoring based on fluorescence quenching of quantum dots using graphene oxide system. Shim et al. [17] reported a chemiluminescence competitive assay for detection of AFB1. Guo et al. [18] developed a sensitive assay employing aptamer as AFB1 recognition probe coupled with real-time quantitative PCR amplification of complementary single-strand DNA.

Surface plasmon resonance (SPR) sensing is a powerful analytical technique for molecular interactions [19-26]. It is relatively challenging to develop SPR sensors for small 
molecules because small molecules cause little change of mass concentration at the sensor surface. Many efforts have been made to improve sensitivity for small molecules by adopting indirect competitive [27], inhibition [28] or sandwich strategies [29]. Dary et al. [30] described a SPR-based immunoassay to the detection of AFB1 using an indirect competitive sensing method. A conjugate consisting of AFB1bovine serum albumin (AFB1-BSA) was attached on sensor chip. Competition between immobilized AFB1-BSA and free AFB1 in solution for binding to an anti-AFB1 antibody leads to decrease of SPR response. Compared to the indirect methods, direct assay shows strength in simplicity, rapidity and reagents-saving. Due to instrumentation improvement, direct SPR assay of small molecules now has been sensitive enough for detection of targets with molecular weight larger than $100 \mathrm{Da}$, e.g. benzoylecgonine, seafood toxin-tetrodotoxin, ochratoxin, and etc. [31-33]. Park et al. [34] reported a direct detection method of AFB 1 by using a SPR immunobiosensor. The genetically engineered GBP-ProG fusion protein enabled self-oriented immobilization of antibody on sensor chip. Under the best loaded density of anti-AFB1 antibodies, the antibody based SPR biosensor allowed detection of AFB1 as low as $1 \mu \mathrm{g} \cdot \mathrm{mL}^{-1}$.

Here we report a direct assay for AFB1 using aptamer based SPR sensor. The aptamer for AFB1 is coated on the SPR sensor chip. The binding of AFB1 on aptamer chip causes SPR signal increase. Then, the bound AFB1 is dissociated from the chip during subsequent flow of binding buffer to regenerate the affinity surface. Under optimized conditions, the aptamer SPR biosensor achieved the selective detection of AFB1 as low as around $0.4 \mathrm{nM}$. It also functioned well in complex sample matrix e.g. diluted red wine and beer sample. This aptamer SPR sensor was able to be reused for many times maintaining good performance, and showed good stability. This aptamer SPR sensor has potential for wide applications.

\section{Materials and methods}

\section{Materials and reagents}

Aflatoxin B1 (AFB1, from Aspergillus flavus), ochratoxin A (OTA, from Aspergilus ochraceus), fumonisin B1 (FB1, from Fuasrium moniliforme), fumonisin B2 (FB2, from Fuasrium moniliforme, structural analog of $\mathrm{FB} 1$ ), zearalenone (ZAE, from Fusarium graminearum) were purchased from Pribolab (Singapore, http://www.pribolab.cn/). Recombinant streptavidin (SA) were purchased from Sangon Biotech (Shanghai, China, http://www.sangon.com/). Working standard AFB1 solutions were freshly prepared from the concentrated stock solution by sequential dilutions in running buffer (10 mM HEPES, $\mathrm{pH} 7.0,20 \mathrm{mM} \mathrm{MgCl}$, $50 \mathrm{mM} \mathrm{NaCl}, 0.1 \%$ Tween-20, $0.3 \%$ acetonitrile). Amine coupling kit (containing 1-Ethyl-3-(3-dimethylaminopropyl)carbodiimide hydrochloride (EDC), N-Hydroxysuccinimide (NHS), 1.0 M Ethanolamine- $\mathrm{HCl}(\mathrm{pH} 8.5)$ ) and $10 \mathrm{mM}$ sodium acetate $\mathrm{pH} 4.5$ were purchased from GE Healthcare (Sweden, http://www3.gehealthcare.cn/). Aptamers were synthesized and purified by Sangon Biotech (Shanghai, China, http://www.sangon.com/). The anti-AFB1 aptamer had the following sequence, $5^{\prime}$-TGG GCA CGT GTT GTC TCT CTG TGT CTC GTG CCC T- $3^{\prime}$, and it was denoted as A34 [12]. The biotinylated aptamer had one biotin labeled at the $5^{\prime}$ terminal of aptamer with a TEG (triethylene glycol) spacer, and it was denoted as A34-5BioTEG. Red wine and beer were purchased from local market. All solutions were prepared using ultrapure water from a Purelab Ultra Elga Labwater system. All other reagents were of analytical grade.

\section{Preparation of the aptamer-coated SPR chip}

In SPR measurements, CM5 sensor chip (GE Healthcare) with four flow cells was used on SPR instrument (BIAcoreT200, GE Healthcare). Aptamer was coated on the SPR chip by using biotinylated aptamer A34-5BioTEG and streptavidin. Streptavidin was first covalently attached on the sensor chip (flow cell 1 and cell 2) using amine coupling method. $10 \mathrm{mM}$ HEPES, pH 7.0, $20 \mathrm{mM} \mathrm{MgCl} 2,0.1 \%$ Tween-20 was employed as running buffer. In detail, the dextran matrix on CM5 sensor chip was first activated with a mixture of $391 \mathrm{mM}$ EDC and $53 \mathrm{mM}$ NHS (1:1, v:v) for $480 \mathrm{~s}$. SA $\left(50 \mu \mathrm{g} \cdot \mathrm{mL}^{-1}\right)$ diluted with acetate buffer ( $\mathrm{pH} 4.5$ ) was passed over the surface for $300 \mathrm{~s}$. Then, $1 \mathrm{M}$ methanolamine ( $\mathrm{pH} 8.5$ ) was used to block the remaining unreacted groups. The flow rate of each step of activation, immobilization, and blocking was set to $10 \mu \mathrm{L} \cdot \mathrm{min}^{-1}$. After that, $10 \mu \mathrm{M}$ A34-5BioTEG diluted with running buffer was injected for $60 \mathrm{~s}$ with a flow rate of $10 \mu \mathrm{L}$. $\mathrm{min}^{-1}$. A34-5BioTEG was only conjugated on the flow cell 2 through the strong interaction between streptavidin and biotin. All buffers were filtered with $0.22 \mu \mathrm{m}$ membrane before use.

\section{Surface plasmon resonance (SPR) assay}

For the detection of AFB1, the reaction temperature was set to $25^{\circ} \mathrm{C}$. The data were processed by Biacore T200 Evaluation Software Version 2.0. Prior to analysis, the whole flow path was primed by running buffer (10 mM HEPES, $\mathrm{pH} 7.0$, $20 \mathrm{mM} \mathrm{MgCl}_{2}, 50 \mathrm{mM} \mathrm{NaCl}, 0.1 \%$ Tween-20, $0.3 \%$ acetonitrile) for three times. Different concentrations of AFB1 were prepared by dilution in running buffer from $1 \mu \mathrm{M}$ AFB1 solution in $0.3 \%$ acetonitrile, and $200 \mu \mathrm{L}$ of solution was prepared for each concentration. The AFB1 solution $(60 \mu \mathrm{L})$ was injected at a flow rate of $30 \mu \mathrm{L} \cdot \mathrm{min}^{-1}$ for $120 \mathrm{~s}$. The binding between AFB1 and aptamer on SPR chip was monitored in real time. After each injection, further running for $250 \mathrm{~s}$ was continued to allow the signal back to the baseline. Report 
points were read at $4 \mathrm{~s}$ in relation to the end of injection in each cycle and AFB1 sample was tested for three times.

\section{Results and discussion}

\section{Design of the SPR sensor and feasibility test}

Scheme 1 shows the principle of direct SPR analysis of AFB1 using aptamer coated chip. Streptavidin (SA) is firstly immobilized on the surface of SPR chip and biotinylated aptamer is then captured through strong interaction between SA and biotin. The binding of AFB1 to the aptamer on the sensor chip causes the changes of mass concentration, leading to significant SPR responses. Therefore, AFB1 detection is finally achieved by monitoring the SPR responses.

A standard amine coupling protocol was used for the immobilization of SA. The dextran matrix on the sensor chip surface was first activated with a mixture of EDC and NHS to give reactive succinimide esters. SA was then covalently attached on the chip through the reaction between uncharged amino groups or other nucleophilic groups of SA and the succinimide esters. Finally, methanolamine was used to block the remaining unreacted ester groups. The sensorgram for the immobilization of SA is shown in Fig. S1A in Electronic Supplementary Material (ESM) with an amount bound of 1921 response unit (RU). The biotinylated aptamer A345BioTEG was then attached on the SPR chip through the strong binding between SA and biotin. As shown in Fig. S1B in ESM, aptamer was efficiently conjugated on the sensor chip with an amount bound of 512 RU.

We then tested the feasibility of SPR analysis of AFB1 with the aptamer coated chip. $25 \mathrm{nM}$ AFB1 was injected at $30 \mu \mathrm{L}$. $\min ^{-1}$ for $120 \mathrm{~s}$. The SPR response increased with the sampling time, and then reached the steady value (Fig. 1). After

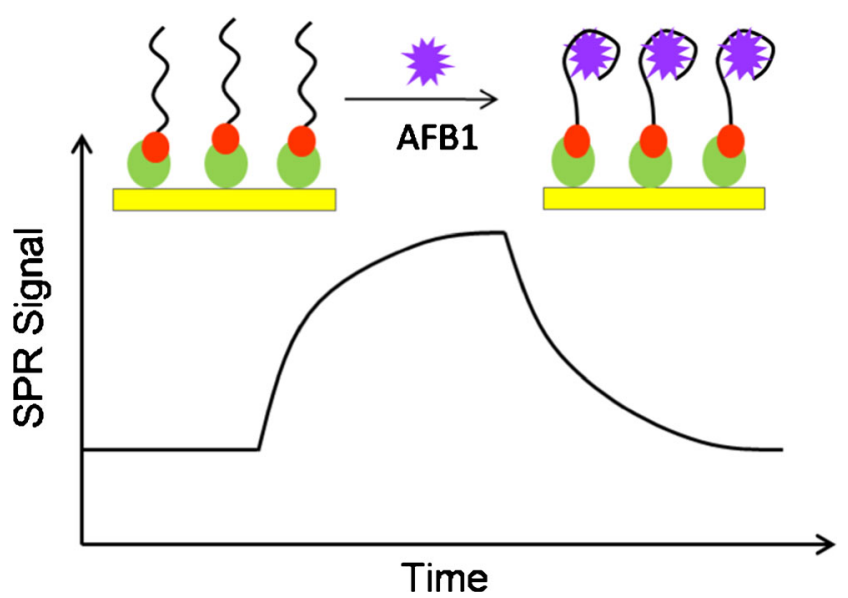

Scheme 1 A schematic illustration for the direct SPR detection of AFB1 using aptamer coated chip

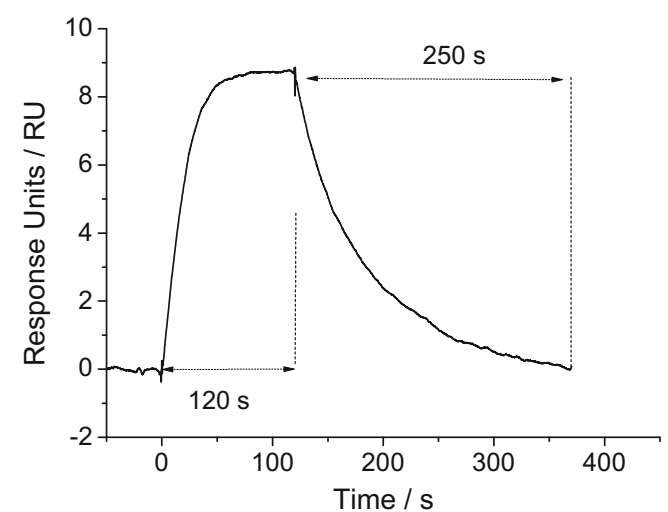

Fig. 1 Feasibility test of direct SPR responses of the association and dissociation of AFB1 $(25 \mathrm{nM})$ in solution on aptamer coated SPR chip

that, SPR response began to drop with further flow of running buffer over the chip. The SPR response returned to the baseline after running for $250 \mathrm{~s}$. The result shows that the AFB1 binds to aptamer on chip with a fast association procedure. In addition, no additional regeneration buffer was needed to regenerate the affinity surface due to fast dissociation of bound AFB1 in the further flow of running buffer. The whole sample procedure was accomplished within $370 \mathrm{~s}$. The result shows it is feasible to develop a direct and rapid analysis of AFB1 using aptamer SPR sensor.

\section{Optimization of method}

The following parameters of running buffer were optimized: (a) $\mathrm{MgCl}_{2}$ concentration and (b) $\mathrm{NaCl}$ concentration. Respective data are given in Fig. S2 in the Electronic Supplementary Material. The concentration of $\mathrm{MgCl}_{2}$ had significant influence on aptamer SPR response to AFB1. When $\mathrm{MgCl}_{2}$ did not exist in the running buffer, no SPR response was observed to AFB1, while the SPR responses to AFB1 increased remarkably with addition of $\mathrm{MgCl}_{2}$ in running buffer. The following experimental conditions were found to give best results: (a) $\mathrm{MgCl}_{2}$ concentration of $20 \mathrm{mM}$ and (b) $\mathrm{NaCl}$ concentration of $50 \mathrm{mM}$. $\mathrm{NaCl}$ was added in running buffer to reduce non-specific adsorption. HEPES buffer $(10 \mathrm{mM}$ HEPES, pH 7.0, $20 \mathrm{mM} \mathrm{MgCl}_{2}, 50 \mathrm{mM} \mathrm{NaCl}, 0.1 \%$ Tween-20, $0.3 \%$ acetonitrile) was chosen as the running buffer.

\section{SPR responses to AFB1}

Under the optimized experimental conditions, direct SPR analysis of varying concentrations of AFB1 was successfully achieved by using the aptamer SPR chip. Time dependent SPR response changes were recorded at different concentrations of AFB1 ranging from $0.0977 \mathrm{nM}$ to $400 \mathrm{nM}$ (Fig. 2). The detection limit of AFB1 was around $0.4 \mathrm{nM}$, and the 

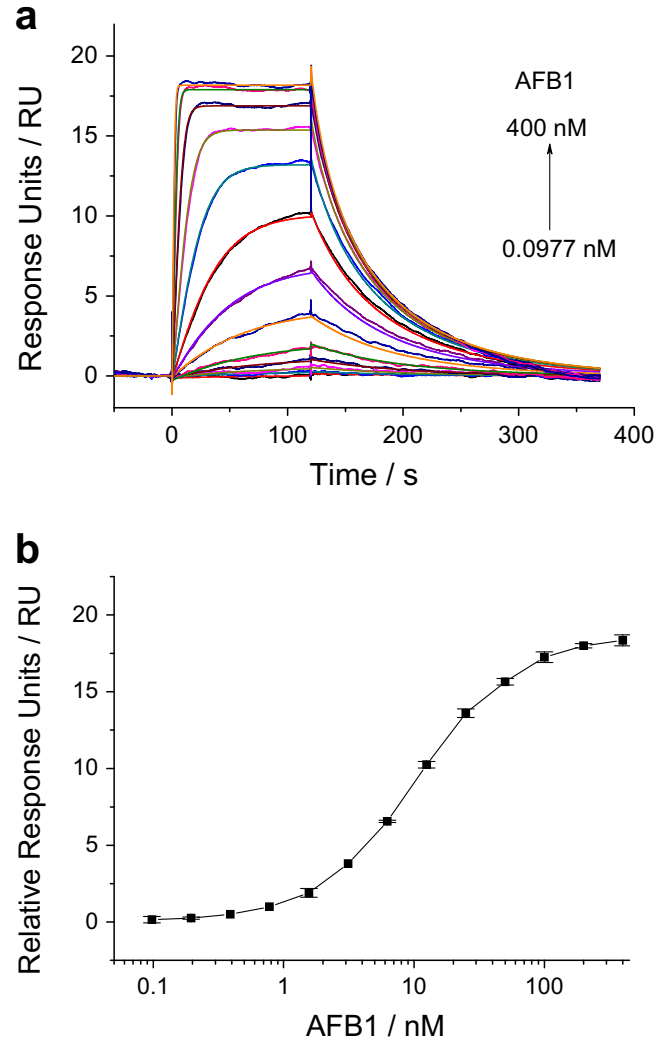

Fig. 2 a Sensorgrams of AFB1 at different concentrations. b Response plot of the aptamer based SPR biosensor for different concentrations of AFB1, data points are the average data \pm standard deviation $(n=3)$. Relative response unit (RU) was used in the calibration plot, and it was obtained by subtracting RU of blank sample from the RU of sample containing AFB1

dynamic range was from $0.4 \mathrm{nM}$ to $200 \mathrm{nM}$. The dissociation constant $\left(K_{\mathrm{d}}\right)$ was determined to be $12 \mathrm{nM}$ by SPR analysis, which is close to that in the previous report [12]. Table 1 summarizes the comparison of a few methods for detection

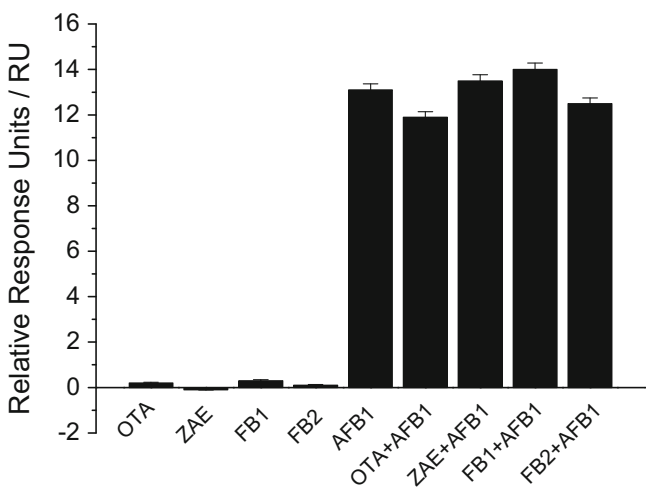

Fig. 3 Specificity test of the aptamer SPR biosensor toward several different mycotoxin samples: OTA, ZAE, FB1, FB2, and AFB1 $(25 \mathrm{nM})$. The concentration of tested mycotoxin was $125 \mathrm{nM}$. Simultaneous presence of AFB1 $(25 \mathrm{nM})$ and other mycotoxin (125 nM each) in sample were also tested, such as OTA + AFB1, $\mathrm{ZAE}+\mathrm{AFB} 1, \mathrm{FB} 1+\mathrm{AFB} 1$, and FB2 + AFB1

of AFB1 using antibodies or aptamers. The sensitivity of our aptamer-based SPR assay is better than that reported antibody SPR assay [30,34], and comparable to that of competitive potentiometric immunoassay [7]. Compared with previous work using the same kind of aptamer with longer sequence (showing similar binding affinity), our detection limit is close to that reported by colorimetric assay based on DNAzyme [16], competitive chemiluminescence assay [17]. However, our detection limit is higher than that of the polymerase chain reaction (PCR) based aptamer assay because PCR allows the remarkable signal amplification [18]. It is also possible to amplify the SPR signals by using some nanomaterials. In addition, the aptamer sensor chip can be reused for many times without significant loss in response activity. After storage at $4{ }^{\circ} \mathrm{C}$ for 80 days, the aptamer sensor chip still showed good response to AFB1 (Fig. S3 in ESM). Compared with other AFB1 detection methods, this direct aptamer SPR sensor is rapid, reusable, and stable.

Table 1 A comparison of some methods for determination of AFB1

\begin{tabular}{|c|c|c|c|c|}
\hline Method & Recognition molecules & $\begin{array}{l}\text { Dynamic range } \\
\text { (nM) }\end{array}$ & $\begin{array}{l}\text { LOD } \\
(\mathrm{nM})\end{array}$ & Reference \\
\hline Antibody based direct SPR assay & antibody & $3 \times 10^{3}-3 \times 10^{5}$ & $3 \times 10^{3}$ & {$[34]$} \\
\hline Antibody based competitive SPR assay & antibody & $9.0-294.0$ & 9.0 & {$[30]$} \\
\hline Fluorescence assay using quantum dots and graphene oxide & aptamer & $3.2-3.2 \times 10^{5}$ & 1.0 & [13] \\
\hline Immunchromatographic strip test using gold nanoparticles & antibody & Not determined & 0.8 & [5] \\
\hline Direct aptamer-based SPR assay & aptamer & $0.4-200$ & 0.4 & this work \\
\hline Competitive chemiluminescence assay & aptamer & $0.3-30$ & 0.3 & [17] \\
\hline Colorimetric assay based on aptamer and split DNAzyme & aptamer & $0.3-3.0 \times 10^{4}$ & 0.3 & {$[16]$} \\
\hline Competitive potentiometric immunoassay & antibody & $0.3-15$ & 0.3 & [7] \\
\hline Flow cytometric immunoassay using upconverting fluorescent nanocrystals & antibody & $0.03-30$ & 0.03 & {$[6]$} \\
\hline Real-time quantitative polymerase chain reaction (PCR) assay & aptamer & $1.5 \times 10^{-4}-15.0$ & $7.5 \times 10^{-5}$ & {$[18]$} \\
\hline
\end{tabular}



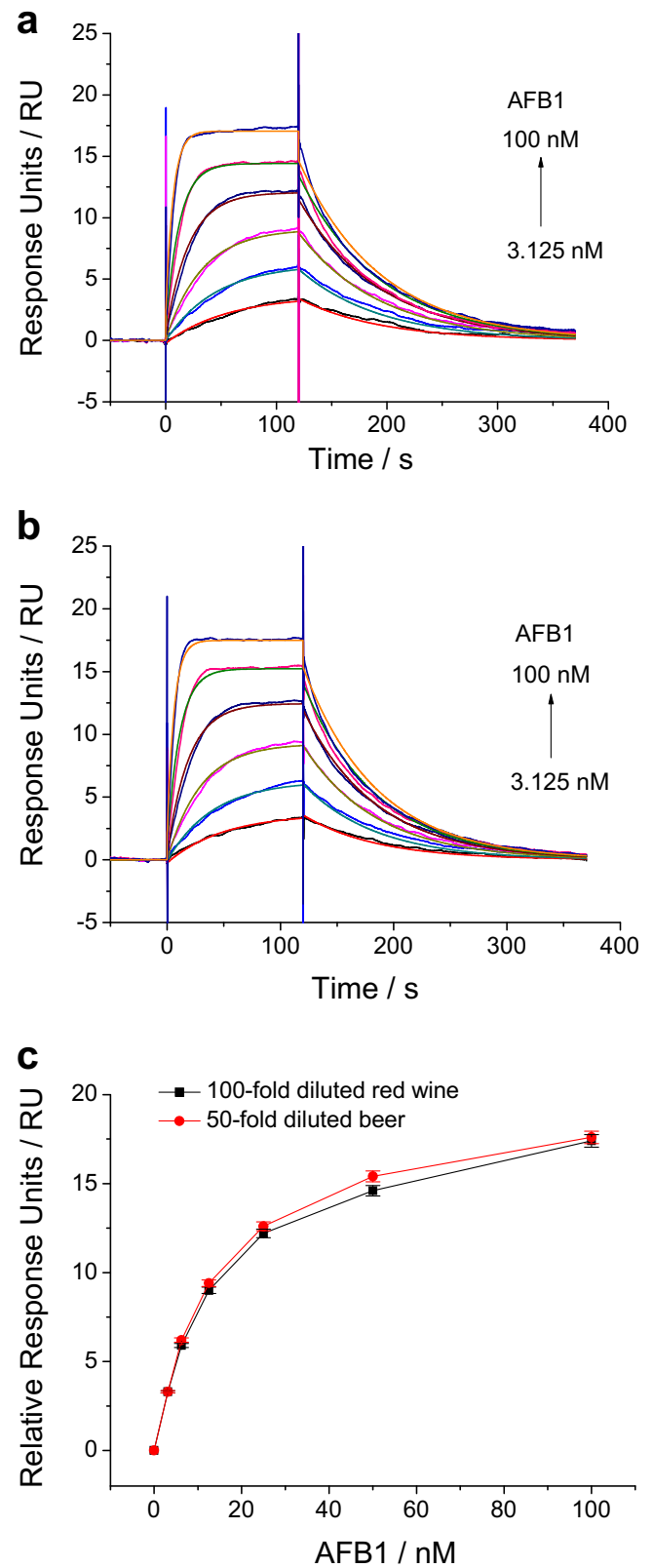

Fig. 4 Sensorgrams of the aptamer based SPR biosensor for detection of AFB1 spiked in 100-fold diluted red wine a or 50-fold diluted beer b. c Response plots of the aptamer based SPR biosensor for AFB1 in 100-fold diluted red wine or 50-fold diluted beer. Relative response unit (RU) was used in the calibration plot, and it was obtained by subtracting RU of blank sample that did not contain AFB1 from the RU of sample containing AFB1

\section{Specificity test}

To evaluate the selectivity of this method, the SPR responses of other mycotoxins were investigated including ochratoxin A (OTA), fumonisin B1 (FB1), fumonisin B2 (FB2), zearalenone (ZAE). These tested mycotoxins did not cause significant SPR responses (Fig. 3). Additionally, the simultaneous presence of these mycotoxins and AFB1 did not result in interference for AFB1 detection. These results indicate this aptamer SPR biosensor has high selectivity for AFB1 detection.

\section{Detection of AFB1 in complex sample matrix}

To further test the feasibility of the method in complex sample matrix, the detection of AFB1 spiked in 100-fold diluted red wine and 50-fold diluted beer were performed. As shown in Fig. 4, the SPR response of different concentrations of AFB1 in 100 -fold diluted red wine and 50 -fold diluted beer is acceptable, which means the aptamer SPR biosensor can be applied to the detection of AFB1 in complex sample matrix. The recovery rates for AFB1 detection in 100-fold diluted red wine and in 50 -fold diluted beer ranged from $87 \%$ to $102 \%$.

\section{Conclusion}

In summary, we developed an aptamer surface plasmon resonance (SPR) sensor for analysis AFB1 in a direct assay format. Aptamer was coated on sensor chip as affinity ligand. The binding of AFB1 on aptamer chip caused significant SPR responses. A fast binding and dissociation process on chip enabled a rapid analysis of AFB1 without requiring additional regeneration steps. This aptamer based SPR sensor allowed the detection limit of AFB1 around $0.4 \mathrm{nM}$. AFB1 spiked in diluted red wine and beer were detected, showing potential for analysis of AFB1 in complex sample matrix. This SPR sensor is stable and reusable. The aptamer SPR sensor integrates the strength of SPR, such as real-time, sensitive and high-throughput detection, and the merits of aptamer in high affinity, stability, and ease of synthesis and labeling. This direct assay format shows advantages over the competitive SPR analysis for AFB1 in simplicity, having potential for applications.

Acknowledgements This work was supported by National Natural Science Foundation of China (Grant No. 21575153, 21435008), Strategic Priority Research Program of the Chinese Academy of Sciences (XDB14030200), and the Key Research Program of the Chinese Academy of Sciences (KFZD-SW-203). We would be grateful to Yuanyuan Chen, Zhenwei Yang (Institute of Biophysics, Chinese Academy of Sciences) for technical help with SPR experiments on Biacore instrument.

Compliance with ethical standards The author(s) declare that they have no competing interests.

\section{References}

1. Brase S, Encinas A, Keck J, Nising CF (2009) Chemistry and biology of mycotoxins and related fungal metabolites. Chem Rev 109(9):3903-3990. doi:10.1021/cr050001f 
2. Minto RE, Townsend CA (1997) Enzymology and molecular biology of aflatoxin biosynthesis. Chem Rev 97(7):2537-2556. doi:10. 1021/cr960032y

3. Han Z, Zheng Y, Luan L, Cai Z, Ren Y, Wu Y (2010) An ultra-highperformance liquid chromatography-tandem mass spectrometry method for simultaneous determination of aflatoxins B1, B2, G1, G2, M1 and M2 in traditional Chinese medicines. Anal Chim Acta 664(2):165-171. doi:10.1016/j.aca.2010.02.009

4. Li P, Zhang Q, Zhang W (2009) Immunoassays for aflatoxins. Trends Anal Chem 28(9):1115-1126. doi:10.1016/j.trac.2009.07.003

5. Di Nardo F, Baggiani C, Giovannoli C, Spano G, Anfossi L (2017) Multicolor immunochromatographic strip test based on gold nanoparticles for the determination of aflatoxin B1 and fumonisins. Microchim Acta. doi:10.1007/s00604-017-2121-7

6. Zhang Y, Liao Z, Liu Y, Wan Y, Chang J, Wang H (2017) Flow cytometric immunoassay for aflatoxin B1 using magnetic microspheres encoded with upconverting fluorescent nanocrystals. Microchim Acta. doi:10.1007/s00604-017-2116-4

7. Li Q, Lv S, Lu M, Lin Z, Tang D (2016) Potentiometric competitive immunoassay for determination of aflatoxin B1 in food by using antibody-labeled gold nanoparticles. Microchim Acta 183(10): 2815-2822. doi:10.1007/s00604-016-1929-x

8. Wu J, Zhu Y, Xue F, Mei Z, Yao L, Wang X, Zheng L, Liu J, Liu G, Peng C, Chen W (2014) Recent trends in SELEX technique and its application to food safety monitoring. Microchim Acta 181(5):479491. doi:10.1007/s00604-013-1156-7

9. Li F, Zhang H, Wang Z, Newbigging AM, Reid MS, Li XF, Le XC (2015) Aptamers facilitating amplified detection of biomolecules. Anal Chem 87:274-292. doi:10.1021/ac5037236

10. Deng B, Lin YW, Wang C, Li F, Wang ZX, Zhang H, Li XF, Le XC (2014) Aptamer binding assays for proteins: the thrombin example-a review. Anal Chim Acta 837:1-15. doi:10.1016/j.aca.2014.04.055

11. Liu X, Zhang X (2015) Aptamer-based technology for food analysis. Appl Biochem Biotechnol 175(1):603-624. doi:10.1007/ s12010-014-1289-0

12. Le LC, Cruz-Aguado JA, Penner GA (2012) DNA ligands for aflatoxin and zearalenone. U.S. patent PCT/CA2010/001292, Sep. 6, 2012

13. Lu ZS, Chen XJ, Wang Y, Zheng XT, Li CM (2015) Aptamer based fluorescence recovery assay for aflatoxin B1 using a quencher system composed of quantum dots and graphene oxide. Microchim Acta 182(3-4):571-578. doi:10.1007/s00604-014-1360-0

14. Wang B, Chen Y, Wu Y, Weng B, Liu Y, Lu Z, Li CM, Yu C (2016) Aptamer induced assembly of fluorescent nitrogen-doped carbon dots on gold nanoparticles for sensitive detection of AFB1. Biosens Bioelectron 78:23-30. doi:10.1016/j.bios.2015.11.015

15. Goud KY, Sharma A, Hayat A, Catanante G, Gobi KV, Gurban AM, Marty JL (2016) Tetramethyl-6-carboxyrhodamine quenching-based aptasensing platform for aflatoxin B1: analytical performance comparison of two aptamers. Anal Biochem 508:19 24. doi:10.1016/j.ab.2016.05.018

16. Seok Y, Byun JY, Shim WB, Kim MG (2015) A structureswitchable aptasensor for aflatoxin B1 detection based on assembly of an aptamer/split DNAzyme. Anal Chim Acta 886:182-187. doi: 10.1016/j.aca.2015.05.041

17. Shim WB, Mun H, Joung HA, Ofori JA, Chung DH, Kim MG (2014) Chemiluminescence competitive aptamer assay for the detection of aflatoxin B1 in corn samples. Food Control 36(1):30-35. doi:10.1016/j.foodcont.2013.07.042

18. Guo X, Wen F, Zheng N, Luo Q, Wang H, Wang H, Li S, Wang J (2014) Development of an ultrasensitive aptasensor for the detection of aflatoxin B1. Biosens Bioelectron 56:340-344. doi:10.1016/ j.bios.2014.01.045

19. Nguyen B, Tanious FA, Wilson WD (2007) Biosensor-surface plasmon resonance: quantitative analysis of small molecule-nucleic acid interactions. Methods 42(2):150-161. doi:10.1016/j.ymeth. 2006.09.009

20. Wang XP, Zhan SY, Huang ZH, Hong XY (2013) Review: advances and applications of surface plasmon resonance biosensing instrumentation. Instrum Sci Technol 41(6):574-607. doi:10.1080/ 10739149.2013.807822

21. Homola J (2003) Present and future of surface plasmon resonance biosensors. Anal Bioanal Chem 377(3):528-539. doi:10.1007/ s00216-003-2101-0

22. Yi X, Hao Y, Xia N, Wang J, Quintero M, Li D, Zhou F (2013) Sensitive and continuous screening of inhibitors of $\beta$-site amyloid precursor protein cleaving enzyme 1 (BACE1) at single SPR chips. Anal Chem 85(7):3660-3666. doi:10.1021/ac303624z

23. Liu L, Xia N, Wang J (2012) Potential applications of SPR in early diagnosis and progression of Alzheimer's disease. RSC Adv 2: 2200-2204. doi:10.1039/c2ra00667g

24. Charlermroj R, Oplatowska M, Gajanandana O, Himananto O, Grant IR, Karoonuthaisiri N, Elliott CT (2013) Strategies to improve the surface plasmon resonance-based immmunodetection of bacterial cells. Microchim Acta 80(7):643-650. doi:10.1007/ s00604-013-0975-x

25. Lei P, Tang H, Ding S, Ding X, Zhu D, Shen B, Cheng Q, Yan Y (2015) Determination of the invA gene of salmonella using surface plasmon resonance along with streptavidin aptamer amplification. Microchim Acta 182(1):289-296. doi:10.1007/s00604-014-1330-6

26. Chen H, Jia S, Gao Y, Liu F, Chen X, Koh K, Wang K (2015) Surface plasmon resonance sensor for norepinephrine using a monolayer of a calix[4]arene crown ether. Microchim Acta 182(9):757-1763. doi:10.1007/s00604-015-1510-z

27. Shankaran DR, Matsumoto K, Toko K, Miura N (2006) Development and comparison of two immunoassays for the detection of 2,4,6-trinitrotoluene (TNT) based on surface plasmon resonance. Sensor Actuat B-Chem 114(1):71-79. doi:10.1016/j.snb. 2005.04.013

28. Choulier L, Nomine Y, Zeder-Lutz G, Charbonnier S, Didier B, Jung ML, Altschuh D (2013) Chemical library screening using a SPR-based inhibition in solution assay: simulations and experimental validation. Anal Chem 85(18):8787-8795. doi:10.1021/ ac4019445

29. Mitchell JS, Wu Y, Cook CJ, Main L (2005) Sensitivity enhancement of surface plasmon resonance biosensing of small molecules. Anal Biochem 343(1):125-135. doi:10.1016/j.ab.2005.05.001

30. Daly SJ, Keating GJ, Dillon PP, Manning BM, O'Kennedy R, Lee HA, Morgan MR (2000) Development of surface plasmon resonance-based immunoassay for aflatoxin B(1). J Agric Food Chem 48(11):5097-5104. doi:10.1021/jf9911693

31. Munoz EM, Lorenzo-Abalde S, Gonzalez-Fernandez A, Quintela O, Lopez-Rivadulla M, Riguera R (2011) Direct surface plasmon resonance immunosensor for in situ detection of benzoylecgonine, the major cocaine metabolite. Biosens Bioelectron 26(11):44234428. doi:10.1016/j.bios.2011.04.056

32. Yakes BJ, Kanyuck KM, DeGrasse SL (2014) First report of a direct surface plasmon resonance immunosensor for a small molecule seafood toxin. Anal Chem 86(18):9251-9255. doi:10.1021/ ac502271y

33. Zhu Z, Feng M, Zuo L, Zhu Z, Wang F, Chen L, Li J, Shan G, Luo SZ (2015) An aptamer based surface plasmon resonance biosensor for the detection of ochratoxin a in wine and peanut oil. Biosens Bioelectron 65:320-326. doi:10.1016/j.bios.2014.10.059

34. Park JH, Kim Y-P, Kim I-H, Ko S (2014) Rapid detection of aflatoxin $\mathrm{B} 1$ by a bifunctional protein crosslinker-based surface plasmon resonance biosensor. Food Control 36(1):183-190. doi:10. 1016/j.foodcont.2013.08.038 